\title{
O Jato de Ar Quente como Estímulo Punidor em Rattus Norvegicus ${ }^{1}$
}

\author{
Marcus Bentes de Carvalho Neto ${ }^{2}$ \\ Thrissy Collares Maestri \\ Gracy Kelly da Silva Tobias \\ Thalita Coutinho Ribeiro \\ Eduarda Cristina Notargiacomo Novaes Coutinho \\ Mariana Moraes Miccione \\ Rita de Cássia Valente Oliveira \\ Fabiane Soraia da Silva Ferreira \\ Danielle Chaves de Farias \\ Daniele Moreira \\ Universidade Federal do Pará
}

\begin{abstract}
RESUMO - Na pesquisa experimental sobre controle aversivo, o choque elétrico tem sido predominantemente utilizado como estímulo punidor. O presente trabalho descreve o uso de um equipamento que emite um jato de ar quente o qual pode ser um estímulo alternativo a ser usado em estudos sobre contingências aversivas. A função punidora do jato de ar quente foi avaliada tanto quando aplicado continuamente (CRF) quanto intermitentemente (FR3) e nos dois casos foi registrada supressão parcial $(98,4 \%$ e $71,15 \%$, respectivamente) da resposta de pressão à barra previamente fortalecida de forma contínua. A supressão foi maior na punição contínua, corroborando os dados com choque elétrico descritos na literatura. O equipamento e o estímulo mostraram-se adequados do ponto de vista técnico e científico e o aparato pode ser uma alternativa atraente do ponto de vista econômico.
\end{abstract}

Palavras-chave: punição; jato de ar quente; estímulos aversivos.

\section{The Hot Air Blast as Punisher Stimulus in Rattus Norvegicus}

\begin{abstract}
In experimental research on aversive control, electric shock has been predominantly used as a punisher stimulus. The present work describes the use of an apparatus that produces a hot air blast which can be an alternative stimulus to be used in research on aversive contingencies. The punishing function of the hot air blast was evaluated in both continuous (CRF) and intermittent (FR3) schedules and, in both cases, a partial suppression $(98,4 \%$ and $71,15 \%$, respectively) of the lever press responding was found, after a history of the continuous reinforcement. Suppression was bigger when the punishing stimulus was applied continuously and these data corroborate the data obtained with electric shock, described in the literature. Both the apparatus and the stimulus seemed to be appropriate from a technical and scientific perspective and the apparatus may become an attractive alternative from a financial standpoint.
\end{abstract}

Key words: punishment; hot air blast; aversive stimuli.

Recentemente, alguns autores (Cameschi \& Abreu-Rodrigues, 2005; Hunziker, 2003; Lerman \& Vorndran, 2002; Perone, 2003; Todorov, 2001; Vollmer, 2002) sugeriram que a área de controle aversivo teria sido prematuramente abandonada na análise do comportamento e que ainda precisaria ser devidamente explorada teórica e empiricamente. $\mathrm{O}$ ponto principal é que o conhecimento atual sobre as relações organismo/ambiente envolvendo contingências aversivas, especialmente na pesquisa básica, seria parcial e insuficiente para compreender o fenômeno em seus múltiplos aspectos.

1 Os autores agradecem as valiosas críticas e sugestões dos Professores Romariz da Silva Barros, Emmanuel Zagury Tourinho, Maria Helena Hunziker, Carlos Barbosa de Souza, Amauri Gouveia Jr, Carla Paracampo e Izabel Florentino. Agradecimentos especiais ao Instituto Evandro Chagas pelo fornecimento dos sujeitos experimentais.

2 Endereço: Rua João Canuto, 510, Centro, Ananindeua, PA, Brasil 67030-130.E-mail:marcus_bentes@yahoo.com.br
Por exemplo, a grande maioria das pesquisas de laboratório foi conduzida com um único tipo de estímulo aversivo: o choque elétrico (Azrin \& Holz, 1966/1975; Baron, 1991; Domjan \& Burkhardt, 1993; Lattal \& Perone, 1998; Sidman, 1989/2001). Os efeitos mais refinados das contingências punitivas em laboratório foram desenvolvidos maciçamente com tal evento, por razões essencialmente práticas (Azrin \& Holz, 1966/1975). Contudo, o choque elétrico possui características muito peculiares (Catania, 1998/1999) e talvez não seja possível estender o conhecimento produzido a partir de seu uso para qualquer outro estímulo aversivo. Haveria, portanto, a necessidade primária de se testar a generalidade dos princípios estabelecidos com a adoção mais ampla e sistemática de diferentes estímulos aversivos. Entretanto, para um estímulo ser útil em condições de laboratório ele precisaria atender a uma série de condições de manipulação que tornassem as medidas padronizadas e confiáveis e a replicação sistemática possível (Azrin \& Holz, 1966/1975), 
exigência esta que restringiu consideravelmente a expansão das investigações para outros estímulos.

Do ponto de vista ético, os novos estímulos também deveriam gerar o mínimo de desconforto e de seqüelas orgânicas e comportamentais aos sujeitos experimentais, mas também deveriam manter, do ponto de vista científico, uma função punitiva suficiente e eficiente para os estudos experimentais com diversos parâmetros e medidas comportamentais (Azrin \& Holz, 1966/1975). Adicionalmente, a aquisição (e manutenção) do equipamento, sendo economicamente viável, permitiria o acesso amplo a esta linha de investigação.

Nos primeiros estudos realizados por Thorndike (1898), basicamente foram usados dois eventos aversivos. Nos estudos com gatos, cães e macacos privados de alimento a própria prisão nas caixas-problema (restrição do espaço físico) funcionava como um estímulo aversivo que era eliminado automaticamente com a saída do aparato. Nos estudos com peixes, por sua vez, a aversão à luz foi usada em procedimentos de fuga para um lado escuro do aquário. Posteriormente, Skinner (1938) usou um dispositivo que desferia tapas na pata dos ratos quando eles pressionavam a barra. Mais tarde, e de forma mais sistemática, alguns estudos usaram o estímulo sonoro como aversivo tanto com sujeitos humanos (Azrin, 1958; Herman \& Azrin, 1964, por exemplo), quanto com pombos (Holz \& Azrin, 1962), gatos (Barry Jr. \& Degelman, 1961) e ratos (Riess, 1970; Knutson \& Bailey, 1974, por exemplo). Outros estímulos aversivos alternativos usados foram: a intensidade luminosa em ratos (F. S. Keller, 1941; J. V. Keller, 1966), alimentos indutores de náuseas pela aplicação de raios-X em ratos (Garcia \& Koelling, 1966; Smith \& Roll, 1967), odor de gatos para ratos (Hubbard \& cols., 2004), rajadas de vento em macacos (Rohles Jr., 1965) e jatos de ar pressurizados em ratos (Ray Jr., 1966) e macacos (Baker \& Ziegelbauer, 1969).

O presente trabalho apresenta como alternativa um equipamento que emite jatos de ar quente, produzidos por um secador de cabelo, como um punidor (Sp) para respostas de pressão à barra (RsPB) em ratos. O equipamento é fácil de obter e de adaptar às caixas de condicionamento operante comumente usadas, com baixo custo financeiro e com parâmetros físicos padronizados. Foram testados inicialmente os efeitos desse evento quando emitido de forma contínua (CRF) e intermitente (FR3). Os objetivos principais do estudo aqui conduzido foram: (a) testar a adequação científica e (b) a viabilidade prática do equipamento para realizar trabalhos sobre controle aversivo em condições de laboratório, tanto para demonstração, quanto para pesquisa básica.

\section{Método}

\section{Sujeitos}

Dois ratos albinos (Rattus norvegicus), machos, com idade, no início do experimento, de 10 meses, obtidos junto ao Instituto Evandro Chagas, com história prévia de modelagem, fortalecimento em CRF e extinção da resposta de pressão à barra (RPB), em privação de água de 48 horas antes de cada sessão experimental e fornecimento contínuo de comida na gaiola-viveiro.

\section{Equipamento e materiais}

O equipamento básico consistiu de uma caixa de condicionamento operante, modelo FUNBEC ${ }^{3}$, adaptada: foi aberto um orifício no teto em formato quadrangular $(6 \times 6 \mathrm{~cm})$ sobre a barra. Sobre o orifício foi colocada uma tela de arame (com quadrados de $0,5 \mathrm{~cm}$ cada), fixada no teto, separando o interior da caixa do secador de cabelo acoplado sobre a caixa de condicionamento. O secador da marca REVLON, modelo RV429AB, operado manualmente, produzia na sua intensidade máxima, ao final de cinco segundos, um aumento de dois graus em relação à temperatura ambiente, com a pressão de $216,5 \mathrm{dyn} / \mathrm{cm}^{2}$ e um nível de ruído de $85 \mathrm{db}$.

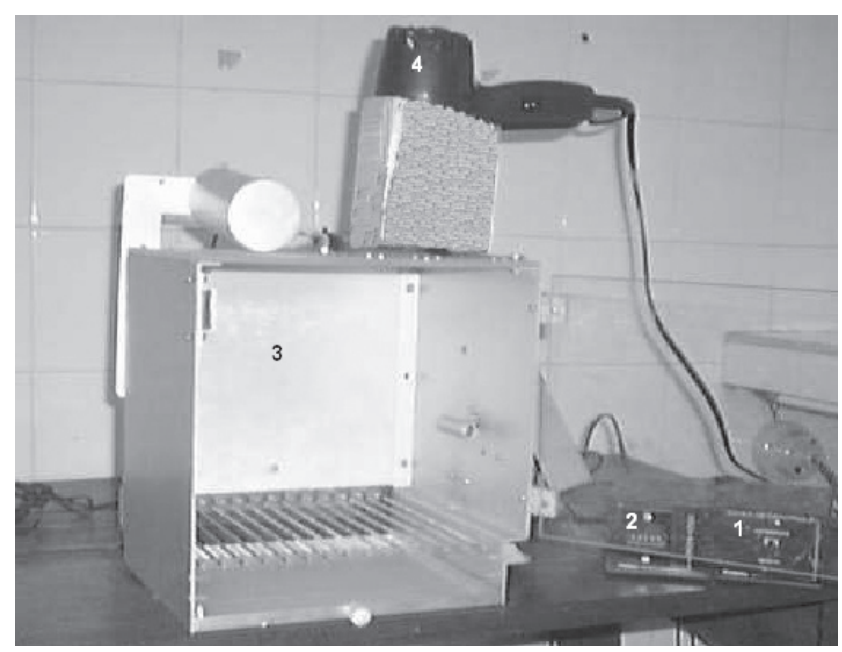

Figura 1. Caixa de condicionamento operante adaptada composta por: caixa de controle do bebedouro; contador numérico; câmara experimental; secador de cabelo (visão geral).

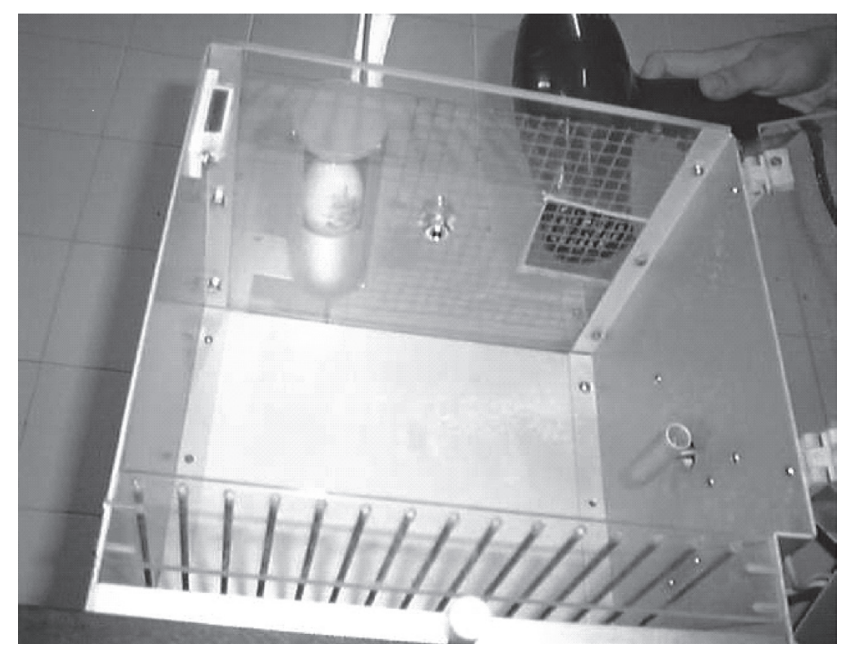

Figura 2. Caixa de condicionamento operante adaptada para pesquisas com jato de ar quente como punidor (visão interna).

3 Qualquer caixa básica de condicionamento operante poderia receber as modificações indicadas e servir como equipamento para estudos sobre contingências aversivas. 


\section{Procedimento}

Os dois sujeitos foram inicialmente expostos a quatro sessões de fornecimento de água em CRF contingente a cada RPB. Em seguida, foram realizadas duas sessões de punição. Para o sujeito 1 vigorou um esquema contínuo de punição e reforçamento positivo (CRF), no qual cada pressão à barra era seguida por um jato de ar quente e por uma gota de água. Para o sujeito 2, vigorou um esquema intermitente de punição (FR3) e contínuo de reforçamento positivo, no qual cada pressão à barra era seguida por uma gota de água e a cada terceira resposta também era produzido um jato de ar quente. $\mathrm{O}$ jato de ar quente era acionado na intensidade máxima do secador por cinco segundos e todas as sessões tiveram uma hora de duração cada.

\section{Resultados}

Para o sujeito 1 (punição contínua), nas quatro sessões iniciais de linha de base foram obtidas as seguintes freqüências totais: 339, 277, 364 e 266 RsPB (311 RsPB em média). As respectivas taxas de respostas por minuto $(\mathrm{R} / \mathrm{min})$ foram: 5,65; 4,61; 6,06 e 4,43 R/min (5.18 R/min em média). Na primeira sessão (punição contínua) obteve-se um total de nove RsPB (queda de 97,11\%). Na segunda sessão obtevese uma RPB (queda de 99,68\%). As respectivas taxas nessa fase foram: 0,15 e $0,01 \mathrm{R} / \mathrm{min}(0,08 \mathrm{R} / \mathrm{min}$ em média). Em média a supressão foi de $98,4 \%$.

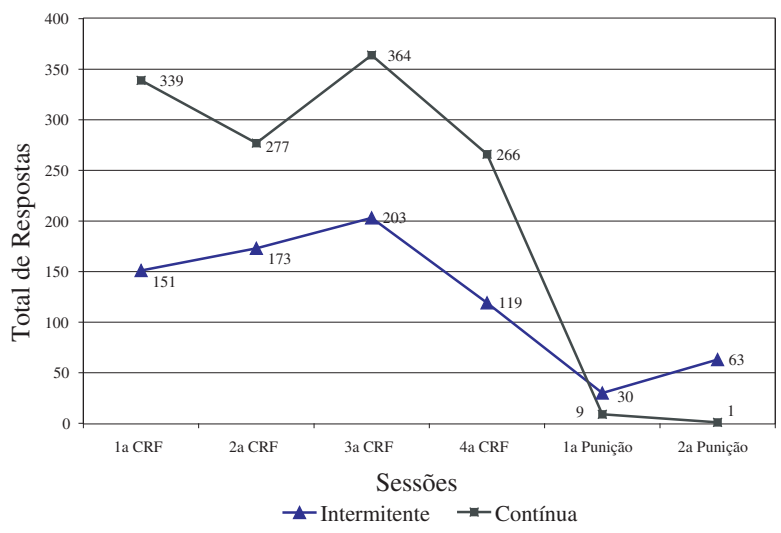

Figura 3. Freqüência absoluta de respostas de pressão à barra ao longo das sessões para os dois sujeitos (punição intermitente e punição contínua).

Para o sujeito 2 (punição intermitente) nas quatro sessões iniciais de linha de base foram obtidas as seguintes frequiências totais: 151, 173, 203 e 119 RsPB (161 RsPB em média). As respectivas taxas de respostas por minuto foram: 2,51 ; 2,88; 3,38 e 1,98 R/min (2.68 R/min em média). Na primeira sessão de punição intermitente, obteve-se um total de 30 RsPB (queda de 81,37\%). Na segunda sessão, obteve-se 63 RsPB (queda de 60,87\%). As respectivas taxas nessa fase foram: 0,5 e 1,05 R/min (1 R/min em média). Em média a supressão foi de $71,12 \%$.

\section{Discussão}

Nas duas condições testadas, o jato de ar quente suprimiu parcialmente o responder. Quando usado de forma contínua a supressão foi mais intensa $(98,4 \%)$ do que quando usado de forma intermitente $(71,12 \%)$. Os dados confirmam os de Estes (1944, Experimento E), comparando punição "periódica" e "aperiódica", usando choque como estímulo punidor em ratos. Um efeito da punição apontado por Azrin e Holz (1966/1975) como sendo geral (quanto menor a razão envolvida entre a resposta e a conseqüência, maior o seu efeito sobre a supressão do responder), mostrou-se aplicável ao jato de ar quente.

Observou-se um aumento na supressão do responder da primeira para a segunda sessão de punição contínua $(2,57 \%)$. O inverso ocorreu com a punição intermitente, quando foi registrada uma recuperação do responder da primeira para a segunda sessão de punição intermitente (20,5\%). Dado o pequeno número de sessões de punição (duas), não é possível avaliar se os dados indicam tendências relacionadas ao tipo de contingência punitiva em vigor em cada caso ou se refletem apenas uma variabilidade inicial na adaptação a nova contingência. Observar os efeitos das duas contingências ao longo de várias sessões poderia elucidar este tópico.

Estão sendo concluídas três pesquisas testando o efeito do jato de ar quente ao longo de 10 sessões de punição contínua e intermitente (Maestri \& Carvalho Neto, 2004), o efeito da ordem de apresentação do esquema de punição, contínuo/ intermitente e intermitente/contínuo (Ribeiro \& Carvalho Neto, 2004) e o efeito da apresentação contínua do jato de ar quente após diferentes histórias de fortalecimento prévio, CRF, VR5 e VI5" (Magalhães \& Carvalho Neto, 2004). Em todas as condições testadas o jato de ar quente, nas especificações físicas aqui descritas e usando um total de 20 ratos, também suprimiu parcialmente o responder, mostrando-se um punidor efetivo nos contextos utilizados.

Outros aspectos também estão sendo estudados pelo grupo. $\mathrm{O}$ jato de ar quente possui ao menos quatro diferentes dimensões que podem funcionar como punidoras: (a) o jato de ar quente em contato com a pele (aquecimento direto); (b) o jato de ar em si (vento na pele); (c) o ruído emitido pelo secador; (d) a elevação da temperatura na caixa (aquecimento indireto). Cada parâmetro está sendo separadamente estudado para que seja possível especificar que dimensão ou dimensões possuem propriedades aversivas. Dados preliminares ainda não publicados indicam que as dimensões (b), (c), (d), separadamente, e (b),(c) combinadas, não seriam capazes de suprimir o responder nas condições aqui descritas. A dimensão (a) parece ser crítica.

Azrin \& Holz (1966/1975) indicam cinco requisitos de um estímulo punitivo "ideal" para estudos em laboratório: (1) Deve possuir uma especificação física precisa; (2) Deve manter contato direto constante com o organismo; (3) Não deveria permitir respostas de fuga não programadas; (4) Deve eliciar poucas reações esqueléticas; (5) Deve permitir o uso de uma ampla gama de valores em um continuиm. $\mathrm{O}$ jato de ar quente possui dimensões físicas especificáveis (1); a resposta selecionada, pressionar uma barra, ocorre exatamente no raio de ação do jato, ou seja, emitir a resposta exige um contato direto com o evento punidor ( 2 e 3); não foram observadas respostas esqueléticas vigorosas (4); é possível manipular a temperatura em uma ampla escala (5). Para estudos sobre punição, portanto, o jato de ar quente atende aos requisitos indicados. Entretanto, para estudos de fuga e esquiva o jato 
de ar quente, na posição em que é usado atualmente, falharia em atender aos requisitos 2 e 3 . Uma caixa de esquiva ativa (similar ao equipamento usado por Ray Jr, 1966), na qual a resposta de pular para o compartimento "seguro" levasse ao fim do contato com um jato de ar quente, acionado de cada extremidade do equipamento de maneira uniforme, seria uma adaptação mais adequada para estes fins.

$\mathrm{O}$ equipamento pode ser montado a partir de qualquer caixa padrão de condicionamento operante e com qualquer secador de cabelos. $\mathrm{O}$ estímulo pode ser apresentado e retirado prontamente e usado a partir de diversos parâmetros (latência, intensidade, esquema). Atualmente foram introduzidas algumas modificações no equipamento. $\mathrm{O}$ teto inteiro foi substituído pela tela de arame. O piso e a barra, originalmente de metal, foram substituídos por equivalentes em acrílico. Um suporte de papelão sustenta o secador de cabelos em uma posição fixa. Com essas alterações a dispersão do calor ficou mais rápida e a precisão do raio de ação do jato também foi melhorada.

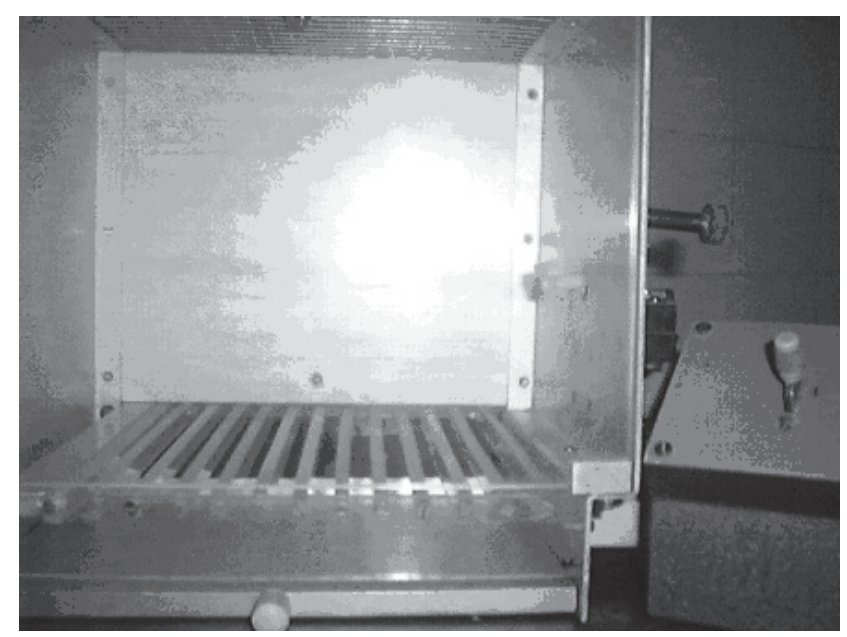

Figura 4. Visão interna da última versão da caixa de condicionamento adaptada, com piso e barra de acrílico e teto inteiramente de tela de metal (visão geral).

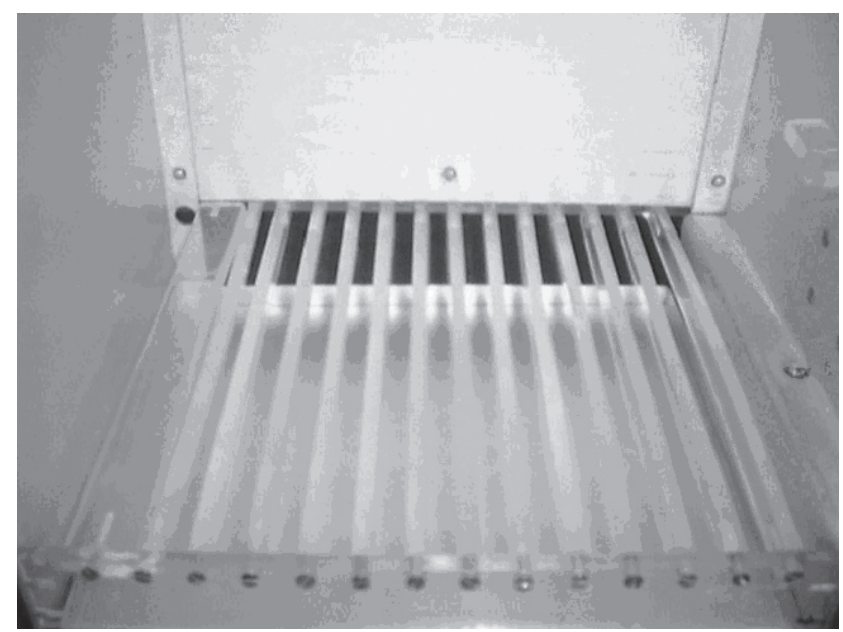

Figura 5. Visão interna da última versão da caixa de condicionamento adaptada, com piso e barra de acrílico e teto inteiramente de tela de metal (visão interna).
Acredita-se que o equipamento possa ser usado tanto para fins didáticos, quanto para a realização de pesquisas experimentais sistemáticas.

\section{Referências}

Azrin, N. H. (1958). Some effects of noise on human behavior. Journal of the Experimental Analysis of Behavior, 1, 183200.

Azrin, N. H. \& Holz, W. C. (1975). Castigo. Em W. K. Honig (Org.), Conducta_operante: investigación y aplicaciones (pp. 455-531) (F. López, E. Galindo \& E. R. Ingesta, Trads). México: Trillas. (Trabalho original publicado em 1966)

Baker, T. W. \& Ziegelbauer, D. (1969). Effect of CS position reversal on extinction in shuttle air-blast avoidance. Psychonomic Science, 17(5), 290-291.

Baron, A. (1991). Avoidance and punishment. Em I. H. Iversen \& K. A. Lattal (Orgs.), Techniques in the behavioral and neural sciences: Experimental analysis of behavior (pp. 95-105). Amsterdam: Elsevier.

Barry Jr., J. J. \& Degelman, J. (1961). A high-power noise amplifier with an electronic keying system. Journal of the Experimental Analysis of Behavior, 4, 85-86.

Cameschi, C. A. \& Abreu-Rodrigues, J. (2005). Contingências aversivas e comportamento emocional. Em J. Abreu-Rodrigues \& M. R. Ribeiro (Orgs.), Análise do comportamento: pesquisa, teoria e aplicação (pp. 112-136). Porto Alegre: Artmed.

Catania, A. C. (1999). Aprendizagem: comportamento, linguagem e cognição. ( $4^{\mathrm{a}}$ ed., D. G. Souza \& cols., Trads.) Porto Alegre: Artmed. (Trabalho original publicado em 1998)

Domjan, M. \& Burkhardt, B. (1993).The principles of learning and behaviour. Pacific Grove: Brooks/Cole.

Estes, W. K. (1944). An experimental study of punishment. Psychological Monographs, 53(3).

Garcia, J. \& Koelling, R. A. (1966). Relation of cue to consequence in avoidance learning. Psychonomic Science, 4, 123-124.

Herman, R. L. \& Azrin, N. H. (1964). Punishment by noise in an alternative response situation. Journal of the Experimental Analysis of Behavior, 7(2), 185-188.

Holz, W. C. \& Azrin, N. H. (1962). Recovery during punishment by intense noise. Psychological Reprints, 11, 655-657.

Hubbard, D. T., Blanchard, D. C., Yang, M., Markham, C. M., Gervacio, A., Chum-I, L. \& Blanchard, R. J. (2004). Development of defensive behavior and conditioning to cat odor in the rat. Physiology \& Behavior, 80, 525-530.

Hunziker, M. H. L. (2003). Desamparo aprendido. Tese de Livre Docência, Universidade de São Paulo, São Paulo.

Keller, F. S. (1941). Light-aversion in the white rat. Psychological Record, 4, 235-250.

Keller, J. V. (1966). Delayed escape from light by albino rat. Journal of the Experimental Analysis of Behavior, 9(6), 655-658.

Knutson, J. F. \& Bailey, M. I. (1974). Free-operant escape-avoidance of noise by rats. Journal of the Experimental Analysis of Behavior, 22(1), 219-229.

Lattal, K. A. \& Perone, M. (Orgs.) (1998). Handbook of research methods in human operant behavior. New York: Plenum.

Lerman, D. C. \& Vorndran, C. M. (2002). On the status of knowledge for using punishment: Implications for treating behavior disorders. Journal of Applied Behavior Analysis, 35(4), 431-464. 
Maestri, T. C. \& Carvalho Neto, M. B. (2004). Os efeitos de uma longa história (10 sessões) de apresentação contínua e intermitente de um jato de ar quente sobre a frequiência de pressão à barra em Rattus norvegicus. [Resumo]. Em Departamento de Psicologia Experimental, Programa de Pós-Graduação em Teoria e Pesquisa do Comportamento, Universidade Federal do Pará (Org.), Caderno de Resumos, VII Semana Científica do Laboratório de Psicologia (pp.25-26), Belém.

Magalhães, P. G. S. \& Carvalho Neto, M. B. (2004). Os efeitos de diferentes histórias fortalecimento operante sobre a sensibilidade a eventos punitivos contínuos (jatos de ar quente). [Resumo]. Em Departamento de Psicologia Experimental, Programa de Pós-Graduação em Teoria e Pesquisa do Comportamento, Universidade Federal do Pará (Org.), Caderno de Resumos, VII Semana Científica do Laboratório de Psicologia (p. 26), Belém.

Perone, M. (2003). Negative effects of positive reinforcement. The Behavior Analyst, 26(1), 1-14.

Ray Jr., A. J. (1966). Shuttle avoidance: rapid acquisition by rats to a pressurized air unconditioned stimulus. Psychonomic Science, 5(1), 29-30.

Ribeiro, T. C. \& Carvalho Neto, M. B. (2004). Alguns efeitos da apresentação contínua e intermitente de um jato de ar quente sobre a freqüência de pressão à barra em Rattus norvegicus. [Resumo]. Em Departamento de Psicologia Experimental, Programa de Pós-Graduação em Teoria e Pesquisa do Comportamento, Universidade Federal do Pará (Org.), Caderno de Resumos, VII Semana Científica do Laboratório de Psicologia (p. 26), Belém.
Riess, D. (1970). The buzzer as a primary aversive stimulus: I. Unconditioned acceleration and summation of conditioned and unconditioned acceleration. Psychonomic Science, 21(3), 167-169.

Rohles Jr., F. H. (1965). Wind as an aversive stimulus. Journal of the Experimental Analysis of Behavior, 8(4), 203-205.

Sidman, M. (2001). Coerção e suas implicações. (M. A. P. Andery e T. M. P. Sério, Trads). Campinas: Livro Pleno. (Trabalho original publicado em 1989)

Skinner, B. F. (1938). The behavior of organisms. New York: Appleton-Century-Crofts.

Smith, J. C. \& Roll, D. L. (1967). Trace conditioning with X-rays as an aversive stimulus. Psychonomic Science, 9(1), 11-12.

Thorndike, E. L. (1898). Animal intelligence: An experimental study of the associative processes in animals. Psychological Review Monograph Supplements, 2(4).

Todorov, J. C. (2001). Quem tem medo de punição? Revista Brasileira de Terapia Comportamental e Cognitiva, 3(1), 37-40.

Vollmer, T. R. (2002). Punishment happens: Some comments on Lerman and Vorndran's review. Journal of Applied Behavior Analysis, 35(4), 469-473.

Recebido em 13.12.2004

Primeira decisão editorial em 25.05.2005

Versão final em 15.11.2005

Aceito em 29.11.2005

\section{CONGRESSO INTERNACIONAL PSICOTERAPIA DE GRUPO}

\section{Grupos: Conectando indivíduos, comunidades e culturas}

Data: 17 a 22 de Julho de 2006

Local: FECAP - São Paulo/SP

Informações e inscrições:

www.eventus.com.br/iagp

iagp2006@eventus.com.br

Telefone: (11) 3361-3056

Fax: (11) 3361-3089 